\title{
Solitons in Relativistic Plasmas by He's Variational Principle
}

\author{
Sami Atif \\ Applied Mathematics Research Center, Department of Mathematical Sciences \\ Delaware State University Dover, DE 19901-2277, USA \\ Daniela Milovic \\ Faculty of Electronic Engineering, Department of Telecommunications \\ University of Nis, Aleksandra Medvedeva 14, 1800 Nis, SERBIA \\ Essaid Zerrad \\ Department of Physics and Pre-Engineering \\ Delaware State University, Dover, DE 19901-2277, USA \\ Anjan Biswas (Corresponding author) \\ Center for Research and Education in Optical Sciences and Applications \\ Department of Mathematical Sciences \\ Delaware State University, Dover, DE 19901-2277, USA \\ E-mail: biswas.anjan@gmail.com
}

The research work of the third author (E.Z.) was fully supported by Army Research Office (ARO) and Air Force Office of Scientific Research (AFOSR) under the award number: W54428-RT-ISP and this

support is thankfully recognized.

The research work of the fourth author (A.B.) was fully supported by NSF-CREST Grant No: HRD-0630388 and Army Research Office (ARO) along with the Air Force Office of Scientific Research (AFOSR) under the award number: W54428-RT-ISP and these supports are genuinely and sincerely appreciated.

\begin{abstract}
The dynamics of electromagnetic solitons in relativistic plasmas is studied in this paper by the aid of He's semi-inverse variational principle. Both Kerr law as well as power law nonlinearity are studied in this paper. The domain restriction of the soliton parameters and the perturbation coefficients are identified.
\end{abstract}

Keywords: Solitons, Plasmas, Variational principle, Integrability

\section{Introduction}

The study of solitons in the context of Langmuir waves in plasmas has been going on for the past few years. During this time frame there has been a considerable amount of progress that has been made. The emission of Langmuir waves in the form of small-scale localized electrostatic bursts have been observed directly in waveform data in many space plasma environments, such as solar wind, auroral region and polar cap. The comparison of observations in various space regions has shown that emissions are seen in associations with warm electron fluxes and have common characterestic properties such as burst-like character, an irregular structure, amplitude variations and a low frequency modulation. Langmuir wave bursts occur in association with electron fluxes with energies $100-400 \mathrm{eV}$ propagating from distant regions of the magnetosphere during magnetic disturbances. The results of bicoherence analysis of wave data have shown that usually the parametric decay process do not play an important role in the formation of Langmuir wave bursts. It has been found that a typical power spectrum width of single burst is about $10 \%$ of the local plasma frequency, which is larger than the width generated by the thermal effect in Langmuir dispersion. Moreover, power spectra have usually a characterestic form with a dent in the upper part. Studies show that these small-scale bursts tend to be correlated 
with the level of the low frequency wave power. Thus in the framework of the electron beam-plasma interaction, the presence of the low frequency turbulence is expected to play a prominent role in the generation of these plasma oscillations. The theoretical model in the quasi-linear statistical approximation has been developed for a beam-plasma instability in the magnetized plasma in the presence of low frequency turbulence. It has been shown that the beat-type waveforms of Langmuir emissions can be explained by interference between waves excited by an electron beam and scattering off the density uctuations. The frequency width of the burst spectrum increases sufficiently due to the resonant wave scattering providing the wave power access to phase space regions with low growth rates [A. P. Misra 2002; S. Samanta, 2007].

Recently relativistic electromagnetic solitons in electron-ion plasmas have been studied in detail analytically, numerically by uid simulations and by multidimensional particle-in-cell simulations. On the other hand, a physically more realistic but also a more complex case of relativistic electromagnetic solitons with a linear polarization was studied in a weak amplitude limit [A. Biswas, 2005]. Thus the theory of solitons is very important in various areas of plasma physics and is an essential componet of plasma turbulence.

\section{Governing equation}

The governing equation for the study of solitons due to Langmuir waves in plasmas is given by the perturbed nonlinear Schr odinger's equation (NLSE). The dimensionless form of the perturbed NLSE is given by [S. Bagchi, 2010; L. Hadzievski, 2002; A. Mancic, 2006].

$$
i q_{t}+a q_{x x}+b F\left(|q|^{2}\right) q=v \frac{q_{x x}^{*}}{|q|^{2}} q^{2}
$$

In (1), $a$ represents the coefficient of the dispersion term, while $b$ is the coefficient of the nonlinear term. Also in the left-hand side the first term represents the evolution term. This equation models the propagation of solitons in relativistic palsmas [L. Hadzievski, 2002; A. Mancic, 2006]. Also, in (1), $F$ is a real-valued algebraic function and it is necessary to have the smoothness of the complex function $F\left(|q|^{2}\right) q: C \mapsto C$. Considering the complex plane $\mathrm{C}$ as a two-dimensional linear space $R^{2}$, the function $F\left(|q|^{2}\right) q$ is k times continuously differentiable, so that [A. P. Misra, 2006]

$$
F\left(|q|^{2}\right) q \in \bigcup_{m, n=1}^{\infty} C^{k}\left((-n, n) \times(-m, m) ; R^{2}\right)
$$

\section{He's variational principle}

In this section, HVP will be introduced. Subsequently, it will be applied to carry out the integration of (1) with the two forms of nonlinearity $F$ in (1). The starting point is the solitary wave ansatz that is given by [A. Biswas, 2010]

$$
q(x, t)=g(s) e^{i \phi},
$$

where $g(s)$ represents the shape of the soliton and

$$
\begin{gathered}
s=x-v t, \\
\phi=-k x+\omega t+\theta,
\end{gathered}
$$

Here, $v$ is the velocity of the soliton, $k$ is the frequency while $\omega$ is the soliton wave number and $\theta$ is the phase constant. Substituting this ansatz into (1) and equating the real and imaginary parts yields respectively the following pair of relations

$$
(a-v) g^{\prime \prime}-\left(\omega+a k^{2}-v k^{2}\right) g+b g F\left(g^{2}\right)=0
$$

and 


$$
v=-2 a k(a+v)
$$

Multiplying both sides of (6) by $g^{\prime}$ and integrating yields

$$
(a-v)\left(g^{\prime}\right)^{2}-\left(\omega+a k^{2}-v k^{2}\right) g^{2}+2 b \int g g^{\prime \prime} F\left(g^{2}\right) d g=K
$$

where $K$ is a constant. The stationary integral $\mathrm{J}$ is defined as

$$
J=\int_{-\infty}^{\infty} K d s=\int_{-\infty}^{\infty}\left[(a-v)\left(\frac{d g}{d s}\right)^{2}-\left(\omega+a k^{2}-v k^{2}\right) g^{2}+2 b \int g g^{\prime \prime} F\left(g^{2}\right) d g\right] d s
$$

Now, the 1-soliton solution ansatz, given by

$$
g(s)=A f\left[\frac{1}{\cosh (B s)}\right]
$$

is substituted into (10). Here, in (11), the parameters $A$ and $B$ represent the amplitude and inverse width of the soliton respectively, and the functional $\mathrm{f}$ is appropriate for the particular non-Kerr law nonlinearity. He's semi-inverse variational principle states that the parameters $A$ and $B$ are determined from the solution of the equations

$$
\frac{d J}{d A}=0
$$

and

$$
\frac{d J}{d B}=0
$$

The parameters $A$ and $B$ will now be determined for the two cases of nonlinearity in the following subsections.

\subsection{Kerr law}

The origin of Kerr law nonlinearity is due to nonlinear response that is related to the non-harmonic motion of bound electrons under the inuence of an applied field. As a result, the induced polarization is

not linear in the electric field, but involves higher order terms in electric field amplitude. In the case of Kerr law nonlinearity where $F(u)=u$, the perturbed NLSE is given by

and therefore (13) reduces to

$$
i q_{t}+a q_{x x}+b|q|^{2} q=v \frac{q_{x x}^{*}}{|q|^{2}} q^{2}
$$

$$
(a-v) g^{\prime \prime}-\left(\omega+a k^{2}-v k^{2}\right) g+b g^{3}=0
$$

Thus, the stationary integral is given by

$$
J=\int_{-\infty}^{\infty}\left[(a-v)\left(\frac{d g}{d s}\right)^{2}-\left(\omega+a k^{2}-v k^{2}\right) g^{2}+\frac{b g^{4}}{2} d g\right] d s
$$

For Kerr law nonlinearity, the appropriate form of the soliton is given by 


$$
g(s)=\frac{A}{\cosh (B s)}
$$

and thus $J$, from (15), simplifies to

$$
J=\frac{2}{3}(a-v) A^{2} B-\frac{2 A^{2}}{B}\left(\omega+a k^{2}-v k^{2}\right)-\frac{2 b A^{4}}{3 b}
$$

The relations (11) and (12) gives the following pair of algebraic equations

$$
\begin{aligned}
& (a-v) \frac{B^{2}}{3}-\left(\omega+a k^{2}-v k^{2}\right)+\frac{2 b A^{2}}{3 b}=0 \\
& (a-v) \frac{B^{2}}{3}+\left(\omega+a k^{2}-v k^{2}\right)-\frac{b A^{2}}{3}=0
\end{aligned}
$$

Solving for $A$ and $B$ from (18) and (19) gives

$$
\begin{aligned}
& A=\sqrt{\frac{2\left(\omega+a k^{2}-v k^{2}\right)}{b}} \\
& B=\sqrt{\frac{\omega+a k^{2}-v k^{2}}{v-a}}
\end{aligned}
$$

From (20) and (21), the relation between the amplitude (A) and inverse width $(B)$ is given by

$$
\frac{A}{B}=\sqrt{\frac{2(v-a)}{b}}
$$

Thus for Kerr law nonlinearity the 1-soliton solution of (13) is given by (16) where the parameters $A$ and $B$ are given by (20) and (21) respectively and the soliton velocity is given by (7). From (20)-(22), it is possible to conclude that the 1-soliton solution to (13) will exist for

$$
\begin{aligned}
(v-a)\left(\omega+a k^{2}-v k^{2}\right) & >0 \\
b\left(\omega+a k^{2}-v k^{2}\right) & >0
\end{aligned}
$$

and

$$
b(v-a)>0
$$

\subsection{Power law}

This law of nonlinearity arises in nonlinear plasmas that solves the problem of small $K$-condensation in weak turbulence theory. For the case of power law nonlinearity, where $F(u)=u^{n}$, the perturbed NLSE

is given by

and therefore (6) reduces to

$$
i q_{t}+a q_{x x}+b|q|^{2 n} q=v \frac{q_{x x}^{*}}{|q|^{2}} q^{2}
$$

$$
(a-v) g^{\prime \prime}-\left(\omega+a k^{2}-v k^{2}\right) g+b g^{2 n+1}=0
$$

Thus, the stationary integral is given by 


$$
J=\int_{-\infty}^{\infty}\left[(a-v)\left(\frac{d g}{d s}\right)^{2}-\left(\omega+a k^{2}-v k^{2}\right) g^{2}+\frac{b}{n+1} g^{2 n+2} d g\right] d s
$$

For power law nonlinearity, the appropriate form of the soliton is given by

$$
g(s)=\frac{A}{\cosh ^{\frac{1}{n}}(B s)}
$$

and thus $J$, from (28), simplifies to

$$
J=\left\{\frac{(a-v) A^{2} B}{n(n+2)}-\left(\omega+a k^{2}-v k^{2}\right) \frac{A^{2}}{B}+\frac{2 b A^{2 n+2}}{(n+1)(n+2) B}\right\} \frac{\Gamma\left(\frac{1}{2}\right) \Gamma\left(\frac{1}{n}\right)}{\Gamma\left(\frac{1}{2}+\frac{1}{n}\right)}
$$

The relations (11) and (12) gives the following pair of algebraic equations

$$
\begin{gathered}
\frac{(a-v) A^{2} B}{n(n+2)}-\left(\omega+a k^{2}-v k^{2}\right)+\frac{2 b A^{2 n}}{n+2}=0 \\
\frac{(a-v) B^{2}}{n(n+2)}+\left(\omega+a k^{2}-v k^{2}\right)-\frac{b A^{2 n}}{(n+1)(n+2)}=0
\end{gathered}
$$

Solving for $A$ and $B$ from (31) and (32) gives

$$
\begin{aligned}
& A=\left[\frac{(n+1)\left(\omega+a k^{2}-v k^{2}\right)}{b}\right]^{\frac{1}{2 n}} \\
& B=n \sqrt{\frac{\omega+a k^{2}-v k^{2}}{v-a}}
\end{aligned}
$$

From (33) and (34), the relation between the amplitude (A) and inverse width (B) is given by

$$
\frac{A^{n}}{B}=\frac{1}{n} \sqrt{\frac{(n+1)(v-a)}{b}}
$$

These relations also lead to the same constraints of the soliton parameters and coefficients as in (23)-(25) for the solitons to exist in the case of power law nonlinearity.

\section{Conclusion}

In this paper, the He's semi-inverse variational principle is used to carry out the integration of the NLSE with perturbation term. Both, Kerr law as well as the power law nonlinearity are studied. In addition to the solutions, the constraint relations between the soliton parameters and the coefficients are determined that poses a restriction on the choice of these parameters.

These results will be used in future to carry out further investigation of the dynamics of plasmas. For example, the quasi-stationary solitons for the perturbed solitary waves will be determined. The quasi-particle theory of the soliton-soliton interaction will also be developed. In addition, the stochasticity will be taken into consideration. Such results will be explored and will be reported elsewhere.

\section{References}

A. Biswas, D. Milovic \& E. Zerrad. (2010). An exact solution for electromagnetic solitons in relativistic plasmas. Physica Scripta, Volume 81, Issue 2, 025506.

A. Biswas. (2005). Soliton perturbation theory for Alfven waves in plasmas. Physics of Plasma, Vol 12, Issue 2, 
022306.

A. Biswas. (2009). Quasi-stationary solitons for Langmuir waves in plasmas. Communications in Nonlinear Science and Numerical Simulation, Volume 14, Issue 1, 69-76.

A. M. Wazwaz. (2004). The tanh method for traveling wave solutions of nonlinear equations. Applied Mathematics and Computation, Volume 154, Issue 3, 713-723.

A. M. Wazwaz. (2006). Exact solutions for the fourth order nonlinear Schrodinger equations with cubic and power law nonlinearities. Mathematical and Computer Modelling, Volume 43, Issues 7-8, 802-808.

A. M. Wazwaz. (2010). Multiple soliton solutions for the (2+1)-dimensional asymmetric Nizhnik-Novikov-Veselov equation. Nonlinear Analysis: Theory, Methods \& Applications, Volume 72, Issues 3-4, 1314-1318.

A. M.Wazwaz. (2005). The tanh and sine-cosine methods for the complex modiffied KdV and the generalized $\mathrm{KdV}$ equations. Computers and Mathematics with Applications, Volume 49, Issues 7-8, 1101-1112.

A. Mancic, L. Hadzievski \& M. M. Skoric. (2006). Dynamics of electromagnetic solitons in a relativistic plasma. Physics of Plasmas, Volume 13, Issue 5, 052309.

A. Mohamadou, F. Ndzana \& T. C. Kofane. (2006). Pulse solution of the modiffied cubic complex Ginzburg-Landau equations. Physica Scripta, Vol 73, 596-600.

A. P. Misra \& A. Roy Chowdhury. (2002). Modulated ion-acoustic wave near criticalness - A new nonlinear Schrodinger equation in a collisional plasma. Fizika A, Vol 11, Number 4, 163-176. 163-176.

A. P. Misra \& A. Roy Chowdhury. (2006). Modulational instability of dust acoustic waves in a dusty plasma with nonthermal electrons and ions. The European Physical Journal D, Vol 39, Number 1, 49-57.

A. P. Misra. A. Roy Chowdhury \& K. Roy Chowdhury. (2004). Electrostatic acoustic models in a self-gravitating complex plasma with variable charge impurities. Physics Letters A, Volume 323, Issues 1-2, 110-121.

A. Ray, D. Ghosh \& A. Roy Chowdhury. (2008). On the chaotic aspects of three wave interaction in a magnetized plasma. Physics Letters A, Volume 372, Issue 32, 5329-5335.

L. Hadzievski, M. S. Jovanovic, M. M. Skoric \& K. Mima. (2002). Stability of one-dimensional electromagnetic solitons in relativistic laser plasmas. Physics of Plasmas, Volume 9, Number 6, 2569-2574.

L. Steno \& O. M. Gradov. (1994). Solitary surface-wave deformation of a plasma boundary. Physics of Plasmas, Volume 1, 2804-2805.

S. Bagchi, A. P. Misra, K. Roychowdhury \& A. Roy Chowdhury. (2009). Nonlinear oscillations in a magnetized dusty plasma with lowtemperature trapped ions. Chaos, Solitons \& Fractals, Volume 40, Issue 2, 758-765.

S. Bagchi, K. Roychowdhury, A. P. Misra \& A. Roy Chowdhury. (2010). On the formation of shock and soliton in a dense quantum dusty plasma with cylindrical geometry. Communications in Nonlinear Science and Numerical Simulations, Volume 15, Issue 2, 275-280.

S. Samanta, A. P. Misra \& A. Roy Chowdhury. (2007). Effect of obliqueness and external magnetic field on the large amplitude solitary waves in dusty plasma. Planetary and Space Science, Volume 55, Issue 10, $1380-1387$.

T. Xu, B. Tian, L-L. Li, X. Lu \& C. Zhang. (2008). Dynamics of Alfven solitons in inhomogenous plasmas". Physics of Plasmas, Volume 15, Issue 10, 102307. 14.

Y. Xiao \& A. Biswas. (2007). Quasi-particle theory of Alfven soliton interaction in plasmas. International Journal of Theoretical Physics, Volume 46, Number 5, 1370-1380. 